This item was submitted to Loughborough's Research Repository by the author.

Items in Figshare are protected by copyright, with all rights reserved, unless otherwise indicated.

\title{
Burning surfaces evolution in solid propellants: a numerical model
}

PLEASE CITE THE PUBLISHED VERSION

PUBLISHER

Professional Engineering Publishing / @ IMECHE

VERSION

VoR (Version of Record)

LICENCE

CC BY-NC-ND 4.0

REPOSITORY RECORD

Szmelter, Joanna, and P. Ortiz. 2019. "Burning Surfaces Evolution in Solid Propellants: A Numerical Model". figshare. https://hdl.handle.net/2134/4693. 
This item was submitted to Loughborough's Institutional Repository (https://dspace.lboro.ac.uk/) by the author and is made available under the following Creative Commons Licence conditions.

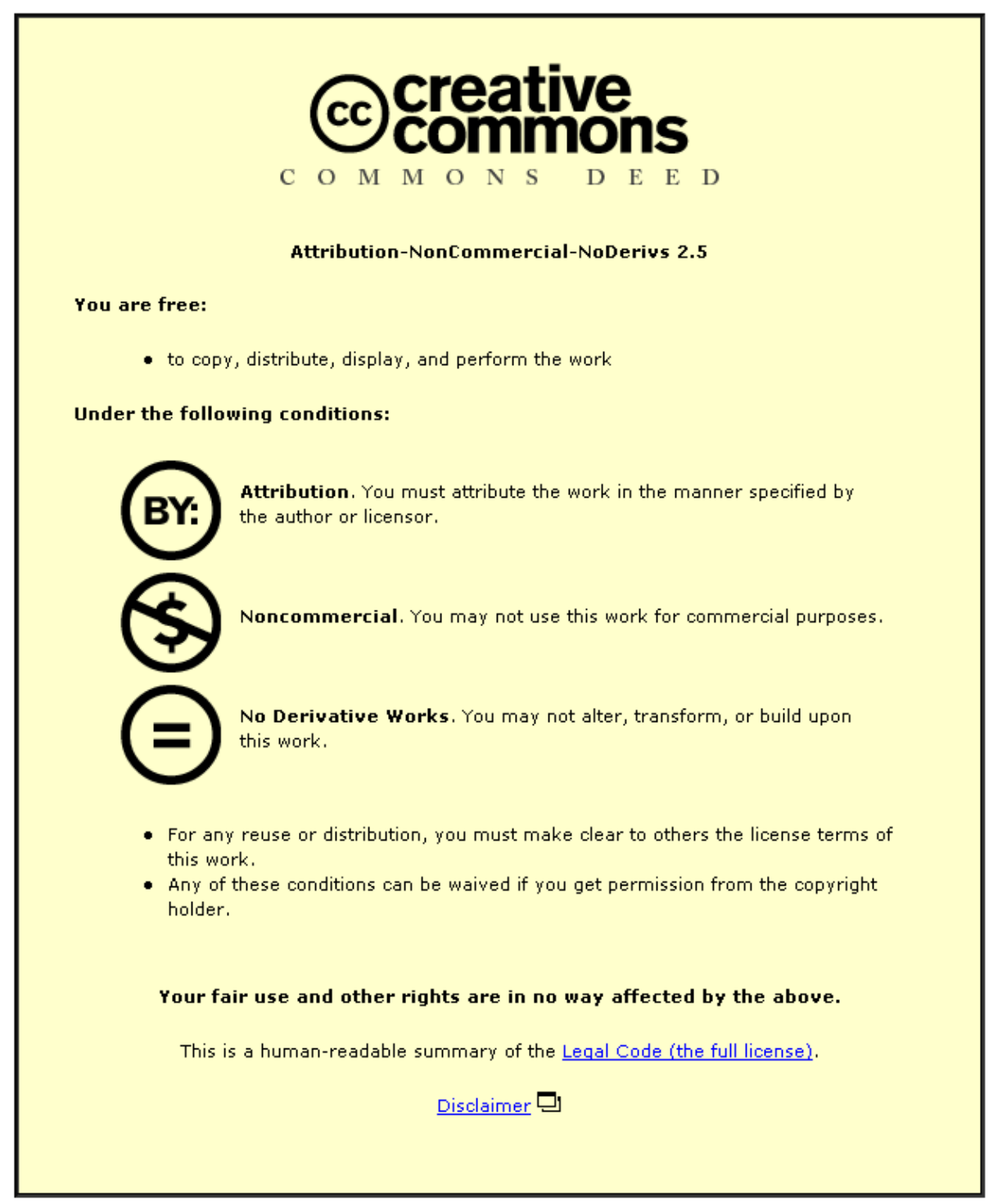

For the full text of this licence, please go to: http://creativecommons.org/licenses/by-nc-nd/2.5/ 


\title{
Burning surfaces evolution in solid propellants: a numerical model
}

\author{
J Szmelter ${ }^{1 *}$ and P Ortiz ${ }^{2}$ \\ ${ }^{1}$ Wolfson School of Mechanical and Manufacturing Engineering, Loughborough University, Leicestershire, UK \\ ${ }^{2}$ University of Granada Ed. Politecnico, Campus Fuentenueva, Granada, Spain
}

The manuscript was received on 22 March 2006 and was accepted after revision for publication on 21 February 2007.

DOI: 10.1243/09544100JAERO102

\begin{abstract}
A methodology for the solution of the internal physics of solid propellant rocket motors is described. The problem involves the simulation of a burning surface - a dynamically changing interface between the solid propellant and combustion gas phases. Burning surfaces can have complex shapes that change in time according to the solid chemistry and deformation, and according to gas parameters. The key element of the proposed model is the development of a new technique to conform the computational mesh to the interface. The paper documents mesh handling and solution procedures suitable for axisymmetric applications. The approach is to treat the problem in a uniform manner for solid and gas phases as a flow with moving sources. Unstructured, dynamically adjusting meshes are employed in the same way for both phases. This paper presents two specific test cases, with non-deforming solids, for which a comparison with theoretical results is possible.
\end{abstract}

Keywords: evolving surfaces, propulsion, unstructured meshes

\section{INTRODUCTION}

For years solid propulsion has been a synonym for compactness, simplicity and cost efficiency. At present, it is used on every stage of small space launchers and in combination with liquid fuel. For tactical missiles solids are employed in every short, medium and many long range units. Although firings of a typical rocket motor during active project development or qualification are costly, numerical simulation is the cheapest and sometimes the only way to understand and predict some phenomena that may be crucial for design. Experimental techniques can handle with difficulty the hot aggressive gases in the combustion chamber, where usually only pressure can be measured. Modelling of the physics involved is very challenging and includes a number of aspects such as: two-phase reactive flow, gas at a wide range of temperatures, multi-species, moving

\footnotetext{
*Corresponding author: Wolfson School of Mechanical and Manufacturing, Loughborouh University, Loughborouh, Leicestershire LE11 3TU, UK. email: j.szmelter@lboro.ac.uk
}

boundaries, fluid/structure coupling, heterogeneous combustion and heat transfer. Currently, research and development of CFD tools for rocket motors are mostly directed towards the understanding of unsteady combustion instabilities. These investigations are challenging per se and frequently have to be limited to simplified problems, e.g. cold flow or sub scale motors, therefore they are usually performed for simple shapes with fixed interfaces. There are a number of publications on combustion instabilities and turbulence modelling for such problems. See reference [1] for a list of representative papers and a state-of-the-art review.

This paper addresses a different aspect of solid propellant rocket motor design. In solid propulsion the design of the charge controls the thrust during flight. The specifications for thrust history may differ substantially between rocket motors depending on the operational requirements of the propelled unit. To improve charge designs it is desirable that the complete operating cycle of the motor from igniter function to blow-down is simulated with reasonably high accuracy. This involves solving for the gas flow in the conduit, the burning of the propellant grain, and the heating and deformation of the solid propellant. 
A number of hydro-codes exist which can be used to examine the deformation and heating of the solid propellant, and there are several gas flow codes suitable for calculating the flow field. However, the coupled problem is more complex, with a moving burning surface at which gas is generated, the motion of which must be calculated. The first published methods for either quasi-static, e.g. [2, 3] or dynamic [4-6], coupling between gas and solid require interfaces with interpolation techniques as well as separate flow solvers and mesh generators for each phase. The latter poses particular demands on the designer, especially in three dimensions, where generation of geometrically complex (e.g. for multi-perforated, multi-fin, charges) surface meshes is difficult. Moreover, from the code development point of view, the need to introduce separate flow solvers with separate data structures, mesh adaptive mechanism, and additional development of complex interfaces between the two phases is an unnecessary complication. In contrast to previous works, it was proposed to treat the problem for gas and solid in an unified way-as a multi-phase flow with the same computational mesh for solid and gas.

As in the Volume of Fluids methods, the proposed technique utilizes a fixed underlying mesh and an independently defined evolving interface. However, in this work a local mesh movement is employed to fit the mesh to the interface, thus ensuring accurate calculation of fluxes on the interface.

In the present development the edge-based data structure is chosen, in the spirit of reference [7]. From the meshing point of view the unstructured edgebased data and the median dual finite volumes offer a high degree of flexibility allowing the scheme to operate on arbitrary polygonal hybrid meshes necessary for complex geometries. Focusing on modelling of the axisymmetric rocket motor it is convenient to use triangular and/or quadrilateral meshes. Triangular meshes are adequate for inviscid flows while quadrilaterals are likely to perform better in the sensitive region of the nozzle throat, particularly when viscous effects are incorporated.

The unstructured data also lends itself well to implementations of different forms of mesh movement, including arbitrary Lagrangian-Eulerian approach, mesh enrichment or remeshing. All of these mesh handling techniques when considered in the modelling of moving interfaces suffer some disadvantages. For example if solely mesh movement is used the mesh would become too distorted. Remeshing, particularly if employed globally, is computationally expensive and requires numerous interpolations affecting the accuracy of the solution. The proposed technique of mesh adjustment aims at alleviating these problems, while maximizing automatic mesh generation to make the scheme user-friendly as a practical engineering tool. Simultaneously, care has been taken to reduce computational costs by using local procedures whenever possible.

The method is presented and is being developed in a general form, which takes into account viscoplastic deformation of solids resulting from highpressure forces. At this stage, the main effort has been directed to demonstrate the flexibility of the mesh adjustment method; therefore the capabilities of the model are shown for the solution of the gas phase in axisymmetric problems.

The remainder of the paper is organized as follows. In the next section the definition of the problem and the governing equations are provided. Section 3 describes the implementation of the finite volume discretization and boundary conditions as well as the mesh adjustment technique. Results and comparisons with theoretical predictions are presented in section 4 . Section 5 concludes the paper.

\section{PROBLEM DEFINITION}

Figure 1 shows a schematic of a solid propellant rocket motor-an axisymmetric high-pressure combustion chamber with solid fuel, before ignition. Following the ignition, when the temperature in the chamber is high enough, the solid starts burning until total burnout.

To highlight that the same equations of the conservation of mass, momentum and total specific energy can be equally used to describe gas phase as well as solid phase, subjected respectively to the burning of the surface and to very high combustion pressure forces, in three dimensions the equations are written in the general, integral form, as

$$
\begin{aligned}
& \frac{\partial}{\partial t} \int_{V} \rho \mathrm{d} V+\int_{S} \rho(\boldsymbol{u}-\boldsymbol{w}) \cdot \boldsymbol{n} \mathrm{d} S=0 \\
& \frac{\partial}{\partial t} \int_{V} \rho \boldsymbol{u} \mathrm{d} V+\int_{S} \rho \boldsymbol{u}(\boldsymbol{u}-\boldsymbol{w}) \cdot \boldsymbol{n} \mathrm{d} S=\int_{S} \tilde{\sigma} \cdot \boldsymbol{n} \mathrm{d} S \\
& \frac{\partial}{\partial t} \int_{V} \rho E \mathrm{~d} V+\int_{S} \rho E(\boldsymbol{u}-\boldsymbol{w}) \cdot \boldsymbol{n} \mathrm{d} S \\
& \quad=\int_{S}(\boldsymbol{q}+\boldsymbol{u} \cdot \tilde{\sigma}) \cdot \boldsymbol{n} \mathrm{d} S
\end{aligned}
$$

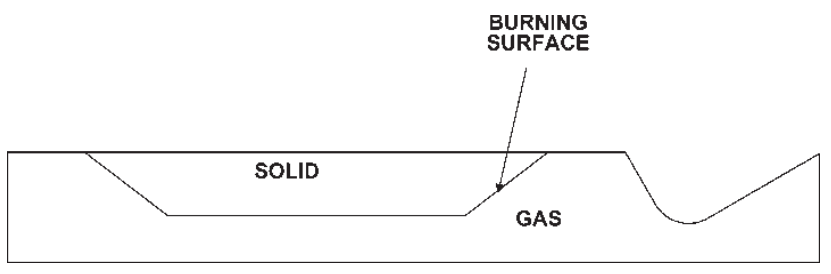

Fig. 1 Definition of the problem. Half of the axisymmetric configuration is shown 
where $\rho$ is the density of the material, $\boldsymbol{u}$ is the velocity, $\boldsymbol{w}$ is the velocity of the moving boundaries (Formally, there is not a moving boundary in this approach, but inclusion of $\boldsymbol{w}$ at this stage is convenient for describing the velocity of mesh movement at discretized level later), $\widetilde{\sigma}$ is the stress tensor, $E$ is the total specific energy, $\boldsymbol{q}$ is the heat flux, $\boldsymbol{n}$ is the unit normal vector pointing outwards from the boundary $S$ of the domain $V$ and $\mathrm{d} S$ symbolizes the surface element. To close the system, stress tensor and equations of state need to be provided in a different form for gas and solid. Here we assume a calorically perfect gas. For the perfect gas the equation of state relates the pressure to total specific energy $E_{\mathrm{g}}$ by

$$
p=(\gamma-1) \rho\left[E_{\mathrm{g}}-0.5\left(u_{x}^{2}+u_{y}^{2}+u_{z}^{2}\right)\right]
$$

where the term $\gamma$ is the ratio of specific heats.

The velocity $\boldsymbol{w}$ of the solid interface depends on the propellant burning rate $r_{\mathrm{b}}$. To define the burning rate we use an empirically derived burning law - a well established simplification in solid propellant combustion. Alternatively, more detailed representations of combustion and surface heat transfer-convective, radiative and solid conduction could be considered, but would dramatically increase the cost of computation. While the detailed combustion modelling is most valuable in a research of physics involved in the solid propellant rocket motors, our choice of using a burning law aims to ensure realistic computational times, essential in design codes.

Among the different forms of burning laws, the simplest is the formula of Vieille. The formula provides a satisfactory approximation for many solid propellants and depends on factors related to the changing pressure in gas and the chemical composition of the solid. The Vieille formula can be written as

$$
r_{\mathrm{b}}=r_{\text {ref }}\left(\frac{p_{\mathrm{c}}}{p_{\text {ref }}}\right)^{n}
$$

where $r_{\text {ref }}$ is a magnitude of the reference burning rate for a given propellant at the reference pressure $p_{\text {ref }}$ (usually $70 \mathrm{Bar}$ ), $p_{\mathrm{c}}$ is the combustion pressure and $n$ the burning rate pressure exponent. When the conservation equations (1) to (3) are solved, the combustion pressure is obtained from the gas state equation (4).

The gas transpiration due to solid combustion contributes to mass, momentum and energy in the form of sources. In order to account for the moving burning surface three types of sources are introduced to the solution. Following general principles presented in reference $[\mathbf{8}]$ values of the transpiration sources are derived from conservation equations ( 1 to 3 ) with the assumption of no mass, momentum and energy flux accumulation on the burning surface $S_{\mathrm{b}}$

$$
\begin{aligned}
& \int_{S_{\mathrm{b}}} \rho_{\mathrm{g}}\left(\boldsymbol{u}_{\mathrm{g}}-\boldsymbol{r}_{\mathrm{b}}\right) \cdot \boldsymbol{n} \mathrm{d} S=-\int_{S_{\mathrm{b}}} \rho_{S} \boldsymbol{r}_{\mathrm{b}} \cdot \boldsymbol{n} \mathrm{d} S \\
& \int_{S_{\mathrm{b}}} \rho_{\mathrm{g}} \boldsymbol{u}_{\mathrm{g}}\left(\boldsymbol{u}_{\mathrm{g}}-\boldsymbol{r}_{\mathrm{b}}\right) \cdot \boldsymbol{n} \mathrm{d} S-\int_{S_{\mathrm{b}}} \widetilde{\sigma}_{\mathrm{g}} \cdot \boldsymbol{n} \mathrm{d} S=-\int_{S_{\mathrm{b}}} \widetilde{\sigma}_{\mathrm{s}} \cdot \boldsymbol{n} \mathrm{d} S \\
& \int_{S_{\mathrm{b}}}\left(\rho_{\mathrm{g}} E_{\mathrm{g}}\left(\boldsymbol{u}_{\mathrm{g}}-\boldsymbol{r}_{\mathrm{b}}\right)-\widetilde{\sigma}_{\mathrm{g}} \boldsymbol{u}_{\mathrm{g}}\right) \cdot \boldsymbol{n} \mathrm{d} S=-\int_{S_{\mathrm{b}}} \rho_{\mathrm{s}} E_{\mathrm{s}} \boldsymbol{r}_{\mathrm{b}} \cdot \boldsymbol{n} \mathrm{d} S
\end{aligned}
$$

where the indexes $g$ and $s$ indicate, respectively, gas and solid phases; the total specific energy $E_{\mathrm{s}}$ corresponds to the solid phase immediately below the interface and it has only the internal energy contribution. Thus, $E_{\mathrm{s}}=C_{\mathrm{p}} T_{\mathrm{f}}$ (Note that $e=C_{\mathrm{v}} T=C_{\mathrm{p}} T_{\text {isochoric }}$. On the burning surface $T_{\text {isochoric }}=T_{\mathrm{f}}$ ), given to the product gases, on the surface $S_{\mathrm{b}}$, is sufficient to raise them to the temperature $T_{\mathrm{f}}$; while $C_{\mathrm{p}}$ is specific heat of products at constant pressure and $T_{\mathrm{f}}$ is the corresponding isochoric flame temperature of propellant. Refer to reference [8] for the in-depth discussion and description of options that can be used for solid-phase treatment. If the viscous effect is negligible, the total stress tensor in the gas phase becomes $\widetilde{\sigma}_{\mathrm{g}}=-p \boldsymbol{I}$.

At this stage of the reported work, the numerical results will be presented for problems without deformation of solids. Moreover, the viscosity of the flow is not taken into account. For inviscid flow, written in integral vector form and using cylindrical coordinates $(x, r)$, with the corresponding notation of $u_{r}$ for the radial and $u_{x}$ for the axial velocities, the conservation equations (1) to (3) reduce to the following form for axisymmetric coordinates

$$
\frac{\partial}{\partial t} \int_{V} \boldsymbol{\Psi} \mathrm{d} V+\int_{S} \boldsymbol{F} \cdot \boldsymbol{n} \mathrm{d} S+\int_{V} \boldsymbol{Q} \mathrm{d} V=0
$$

where inviscid flux vectors $\boldsymbol{F}=\left(\boldsymbol{F}_{x}, \boldsymbol{F}_{r}\right)$, are given by

$$
\begin{gathered}
\boldsymbol{\Psi}=\left\{\begin{array}{c}
r \rho \\
r \rho u_{x} \\
r \rho u_{r} \\
r \rho E
\end{array}\right\}, \quad \boldsymbol{Q}=\left\{\begin{array}{l}
0 \\
0 \\
p \\
0
\end{array}\right\} \\
\boldsymbol{F}_{x}=\left\{\begin{array}{c}
r \rho\left(u_{x}-w_{x}\right) \\
r\left(\rho u_{x}\left(u_{x}-w_{x}\right)+p\right) \\
r\left(\rho u_{r}\left(u_{x}-w_{x}\right)\right) \\
r\left(\rho E\left(\left(u_{x}-w_{x}\right)+p u_{x}\right)\right)
\end{array}\right\}, \\
\boldsymbol{F}_{r}=\left\{\begin{array}{c}
r\left(u_{r}-w_{r}\right) \\
r\left(\rho u_{x}\left(u_{r}-w_{r}\right)\right) \\
r\left(\rho u_{r}\left(\left(u_{r}-w_{r}\right)+p\right)\right) \\
r\left(\rho E\left(u_{r}-w_{r}\right)+p u_{r}\right)
\end{array}\right\}
\end{gathered}
$$


For non-deforming solids $\sigma_{\mathrm{s}} \cdot \boldsymbol{n}=0$, and the corresponding transpiration sources (6) take the form

$$
\begin{aligned}
& \int_{S_{\mathrm{b}}} r \rho_{\mathrm{g}}\left(\boldsymbol{u}_{\mathrm{g}}-\boldsymbol{r}_{\mathrm{b}}\right) \cdot \boldsymbol{n} \mathrm{d} S=-\int_{S_{\mathrm{b}}} r \rho_{\mathrm{s}} \boldsymbol{r}_{\mathrm{b}} \cdot \boldsymbol{n} \mathrm{d} S \\
& \int_{S_{\mathrm{b}}} r\left(\rho_{\mathrm{g}} \boldsymbol{u}_{\mathrm{g}}\left(\boldsymbol{u}_{\mathrm{g}}-\boldsymbol{r}_{\mathrm{b}}\right)-\widetilde{\sigma}_{\mathrm{g}}\right) \cdot \boldsymbol{n} \mathrm{d} S=0 \\
& \int_{S_{\mathrm{b}}} r\left(\rho_{\mathrm{g}} E_{\mathrm{g}}\left(\boldsymbol{u}_{\mathrm{g}}-\boldsymbol{r}_{\mathrm{b}}\right)-\widetilde{\sigma}_{\mathrm{g}} \boldsymbol{u}_{\mathrm{g}}\right) \cdot \boldsymbol{n} \mathrm{d} S \\
& =-\int_{S_{\mathrm{b}}} r \rho_{\mathrm{s}} C_{\mathrm{p}} T_{\mathrm{f}} \boldsymbol{r}_{\mathrm{b}} \cdot \boldsymbol{n} \mathrm{d} S
\end{aligned}
$$

\section{NUMERICAL APPROACH}

\subsection{Spatial and time discretization}

Equation (7) is discretized in space on hybrid (polygonal) meshes with unstructured edge-based data. Here, the median dual finite volume discretization described in detail in reference [9] is used. The edge-based data structure allows for relatively straightforward extensions to three dimensions, is compact, and computationally efficient, while the median dual finite volumes are reported [10] to retain better accuracy than other finite volume arrangements traditionally used for unstructured meshes. By design, the approach constructs faces $S_{j}$ of the dual mesh that bound the control volume $V_{i}$ containing the vertex $i$. The control volume joins the centres of surrounding polygons and centres of the $j=1, l(i)$ edges connecting vertex $i$ with its $l(i)$ neighbours - see Fig. 2 for schematics in two dimensions.

The resulting discretized equations are

$$
\frac{\partial}{\partial t} \boldsymbol{\Psi}_{i}=\mathrm{RHS}_{i}
$$

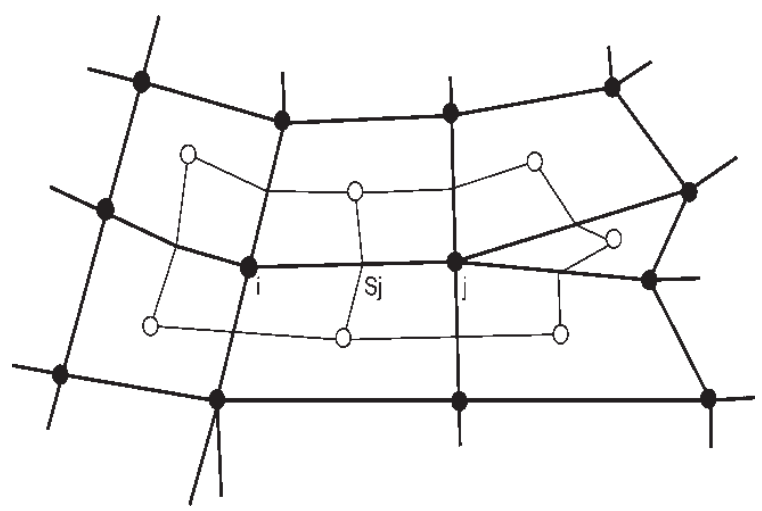

Fig. 2 The edge-based median-dual approach: the edge connecting vertices $i$ and $j$ pierces the face $S_{j}$ of the two-dimensional computational cell surrounding vertex $i$ where the right hand side is

$$
\mathrm{RHS}_{i}=\frac{1}{V_{i}}\left(\sum_{j=1}^{l(i)} \boldsymbol{F}_{j} \mathrm{~d} S_{j}+\boldsymbol{Q}_{i} V_{i}+\boldsymbol{D}_{i}\right)
$$

and $\boldsymbol{\Psi}_{i}$ indicate discretized variables, $\boldsymbol{F}_{i}$ fluxes and $\boldsymbol{Q}_{i}$ source. $S_{j}$ refers both to the median-dual cell face per se and its surface area. The values of fluxes at faces $S_{j}$ are obtained as an average of fluxes calculated in points $i$ and $j$, resulting in a second order centered in space algorithm. For stability of such a scheme the blend $\boldsymbol{D}_{i}$ of second and fourth-order artificial dissipation terms [11] is added. Further, the multi-stage Runge-Kutta discretization in time is employed

$$
\boldsymbol{\Psi}_{i}^{n+1}=\boldsymbol{\Psi}_{i}^{k}-\alpha \delta t \mathrm{RHS}_{i}^{k}
$$

where $n$ is the time level, $\delta t$ is the time step, $k=3$ is the number of Runge-Kutta stages and $\alpha$ is a coefficient with the values of $0.6,0.6$ and 1.0 for the three-stage scheme used in this work.

In practice the transpiration sources (6) are added to the flux terms contributing to the finite volumes assigned to the points at the burning surface. Further, as this problem involves moving meshes, care needs to be taken that the movement does not affect the flow field. In the implementation, the velocity of the control volume faces has to be consistent with the rate of the control volume change, and the calculation of fluxes is conducted in a way that fulfils the volume and the surface geometric conservation laws [12].

\subsection{Boundary conditions}

For walls the free-slip condition is assumed. At the symmetry axis $(r=0)$, values of flow parameters are linearly extrapolated from the surrounding cells. Moreover, for the boundary points the fluxes in (8) are made zero on the wall and are computed as one-sided contribution for other types of boundaries.

The problem associated with specification of boundary conditions at the nozzle exit is well recognized in solid propellant rocket motors. Complication comes from the fact that as shown in Fig. 3 during the burning process the inflow of gases into the nozzle is possible. This situation excludes the simplest treatment by extrapolation of variables. Extrapolation, can be applied for computations where the flow in the nozzle is known a priori to be always the

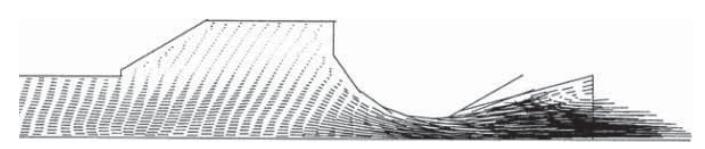

Fig. 3 Velocity vectors showing recirculation of the flow at the nozzle exit 
supersonic outflow at the exit, as would be the case for some steady-state computations. Therefore, for the exit, we have implemented two alternative treatments. First approach it to extend the computational field beyond the nozzle to include external flow and to impose boundary conditions at far field. The far field conditions are specified from the free-stream values or extrapolated from Riemann invariants, depending on the character of the flow-super or subsonic and inflow versus outflow. The extension of the computational field is needed if a detailed history of pressure in the combustion chamber is required prior to the flow choking at the throat or if the flow in the divergent part of the nozzle needs to be computed as it may be needed in support of nozzleless rocket motor designs. However, the extension of the computational field substantially increases the cost of calculations. For problems, when the internal flow in the combustion chamber is of primary interest we follow references [13] and [14] and limit the computational field at the nozzle exit and apply a simple prescription of pressure. Although, from the numerical point of view the prescription of the exit pressure is formally not well posed if the exit is supersonic outlet, the physics of nozzles dictates that the supersonic outlet can only be achieved if the flow reaches a critical condition at the throat. After the flow in the nozzle is choked the flow in the combustion chamber becomes insensitive to flow parameters at the divergent part of the nozzle. A simple numerical check confirms this since the specification of substantially different values of the exit pressure will not change the value of the pressure in the combustion chamber from the moment the nozzle is choked. The prescribed exit pressure must be low enough to allow for the flow to choke. In the examples presented here, the value of pressure is computed numerically from the Mach number-area and isentropic flow relations according to nozzle flow theory (e.g. see reference [15]). Such boundary condition allows for the flow to choke and gives correct flow values in the combustion chamber, however, the values in the divergent part of the nozzle are not physical as the divergent part of the nozzle serves only to numerically establish the critical condition at the throat.

In the special case, when the computational domain for solid is neglected, the moving interface can be viewed as a moving boundary on which influx is prescribed according to the definition of sources in equation (8).

\subsection{Mesh adjustment technique}

This section presents a key development of the work - a combination of locally moving and updating meshes that allows for changes of burning surface. Although, the method is demonstrated for modelling of rocket motors its principles are more general. The same technique can be applied for modelling other evolving interfaces and for problems involving moving rigid bodies. The proposed mesh adjustment algorithm consists of two stages - preparation of the initial geometry and connectivities before the propellant is ignited, and modelling of evolving burning surface. The second stage is used repetitively. All mesh changes performed in the second stage are local and take place only near the burning surface, making the method efficient.

\subsubsection{First stage-preparation of the initial geometry and connectivities}

First an initial mesh is generated for the whole computational domain without distinction between the phases. For generation of triangular meshes the Advancing Front Technique [16] is used. The algorithm repeatedly returns to the initial mesh; therefore in the further description it is referred to as an underlying mesh. Both coordinates and connectivity (defined in terms of the edge data structure) of the underlying mesh are stored.

Independently, a set of points describing the initial shape of the solid surface is supplied as shown schematically in Fig. 4(a). The segments joining the points define the shape of the interface between the solid and gas phases. Therefore, it is important that a sufficient number of points is used to ensure the fidelity of the geometry. The segments also provide the connectivity information of the interface, i.e. for every interface point its neighbouring points are known.

Next, nodes of the mesh positioned at the closest distance to the interface are identified and stored according to procedures described in reference [17]. These nodes are then moved to fit the interface, taking the shortest distance between the initial position of the node and the interface. Note that such movement is moderate since it cannot exceed the size of the mesh element. Consequently, the mesh conforms to the interface as schematically illustrated in Fig. 4(b). In order to retain a good quality of the overall mesh the remaining nodes are also moved using the spring analogy technique [18]. The spring analogy procedure adopted in this work follows closely the detailed description on page 650 of reference [19]. There are several alternative mesh movement techniques that can be implemented (see reference [20] for a recent review).

Following the mesh movement, the interface definition and connectivity are updated. The burning face areas, i.e. in two dimensions, a sum of two semi-lengths of segments associated with each point on the interface, are calculated. Further, the normal to the interface at that point is found using a weighted 

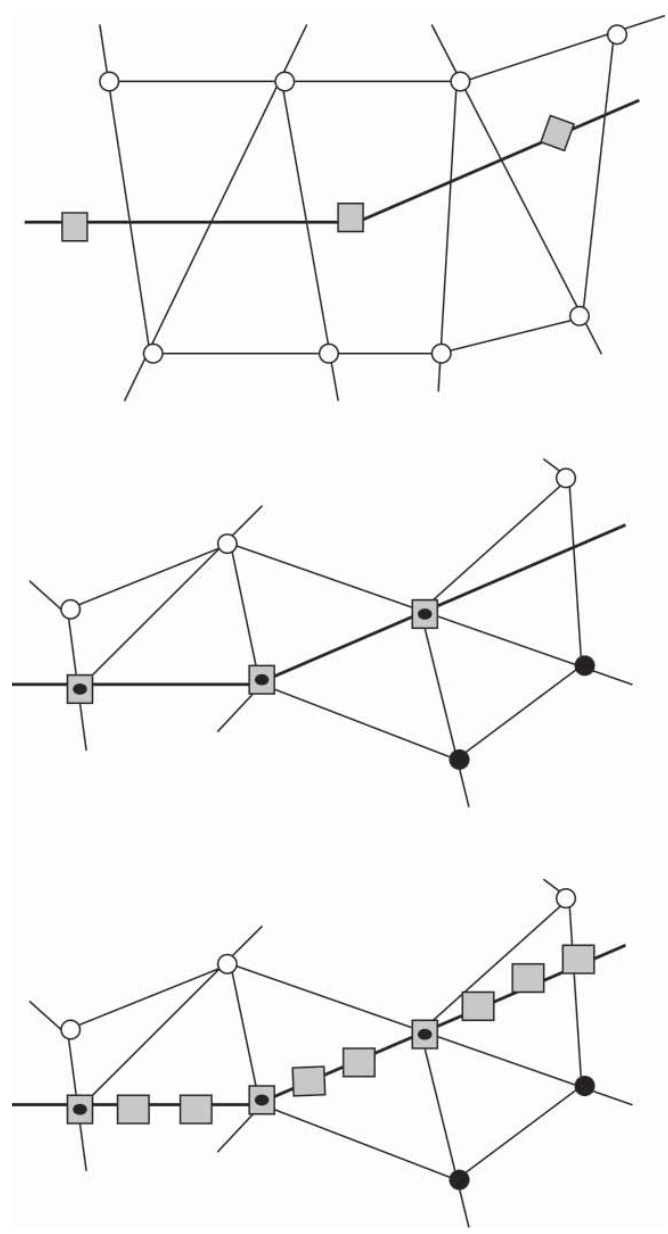

Fig. 4 Mesh adjustment procedure: (a) (top) initial mesh and an independently defined interface, before the solid and gas nodes are recognized; (b) (middle) moved mesh conforming to the interface; (c) (bottom) refined definition of the interface in preparation for the next search. Rectangles indicate points on the interface, filled circles-points in the gas and open circles-points in the solid

average of normal vectors obtained for each interface segment containing this point.

Finally, a topological global check for the whole mesh is performed in order to automatically separate parts of the computational domain belonging to the gas and solid phases, marked in Figs 4(b) and (c). Normal directions to the interface and computational domain boundaries define uniquely sub-domains that correspond to the different phases and are examined so that different flags can be used to mark the following types of edges in the mesh:

(a) edges with two nodes belonging to the same phase;

(b) edges with two nodes belonging to the interface or the boundary; (c) edges with only one node on the interface or the boundary.

This information permits to distinguish between different properties of material (i.e. gas and solids) and to activate different state equations. Most of all, the flagging of the edges is necessary to reduce further searches to much more efficient local operations. When the preparation is completed, the next stage - the modelling of evolving burning surface is activated.

\subsubsection{Second stage-modelling of evolving burning surface}

The mesh nodes now conform to the interface and the contribution of the mass, momentum and energy source fluxes (8) can be evaluated accurately for the median dual finite volumes.

After a time iteration of the flow solution (10), the pressure is updated and the burning velocity of the solid can be computed. In the examples presented, we assumed that the solid is rigid, therefore the coordinates of the new position of the moving interface are obtained from the burning rate (5), proceeding in the direction normal to the interface

$$
\begin{aligned}
x_{\text {new }} & =x_{\text {old }}+\left(r_{\mathrm{b}}\right)_{x} \delta t \\
r_{\text {new }} & =r_{\text {old }}+\left(r_{\mathrm{b}}\right)_{r} \delta t
\end{aligned}
$$

where $x$ and $r$ are the cylindrical coordinates and $\left(r_{\mathrm{b}}\right)_{x}$ and $\left(r_{\mathrm{b}}\right)_{r}$ are the corresponding components of the burning rate. For a deforming propellant this step needs to be modified such that the computation takes into account the velocity of the solid deformation. A limiter defined by the intersection of the boundary - the rocket motor case, and the new position of the interface needs to be applied to ensure that none of the interface points is artificially moved outside the casing. As the propellant burns, the interface moves into the solid domain until total burnout, when only gas is in contact with a standard solid wall (i.e. casing).

If the movements of the burning surface are very small the mesh can be moved without the spring analogy technique. This is repeated for several time iterations. Further, to prevent distortion of the mesh the spring analogy is employed again. The procedure can continue until the distortion of the mesh becomes unacceptable. Then, in preparation of the next search the points defining the interface are added or removed as shown schematically in Fig. 4(c) (addition and removal of points is simplified by the use of unstructured data in the interface definition). Now, the algorithm returns to the underlying mesh stored at the preparation stage and finds the nodes of the underlying mesh, closest to the interface. Unlike in the preparation stage of the scheme, this 

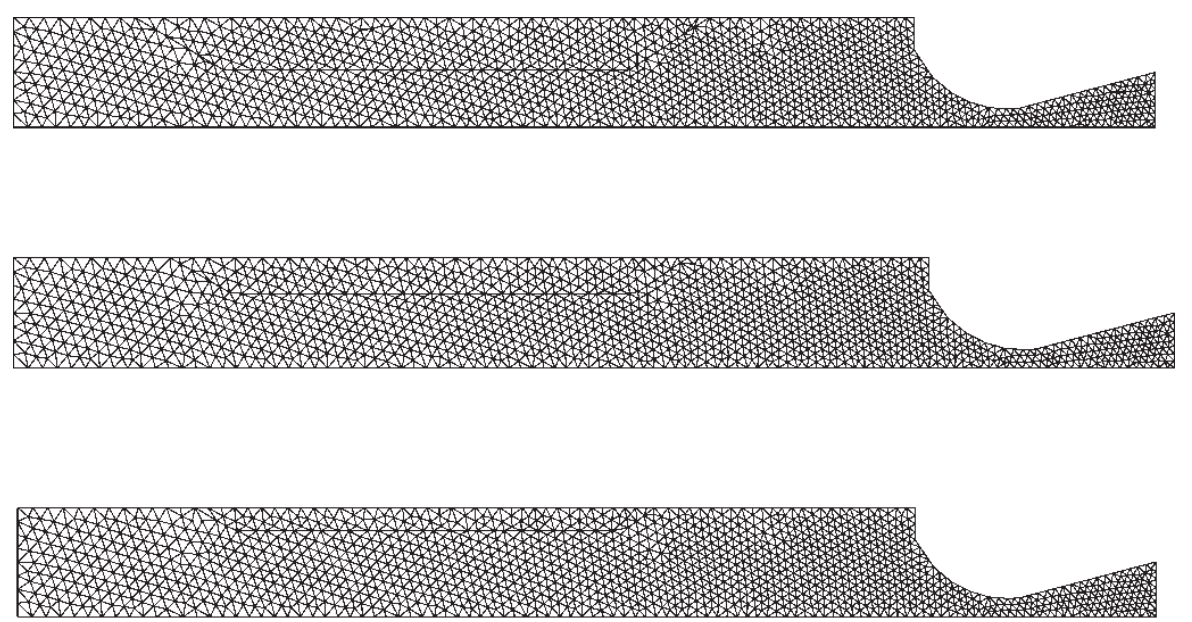

Fig. 5 The illustration of the mesh adjustment technique: the initial mesh after adjustment to the initial shape of the burning surface (top), and the example of two subsequent intermediate meshes (middle and bottom). For clarity, purposely coarse meshes are shown

search is local and fast since it needs only to use information from the edges marked as type $b$ and $c$. The phases are already separated and marked with the edges of type $a$. As in the preparation stage the mesh points are moved again to fit the burning surface. The flags for edges and the definition of the burning surface are updated locally. When the interface moves, mesh nodes previously belonging to the solid phase, enter the domain of the gas phase, while values of flow parameters at these nodes are obtained by linear extrapolation from the neighbouring cells.

This completes the second stage. The stage is repeated until the whole solid is burned out. The numbers of points defining the interface reduces until there are no points (therefore no solid) left. The procedure is illustrated in Fig. 5, where the initial and intermediate meshes are shown for a selected part of the domain. For clarity, a coarse mesh is shown.

For special cases the algorithm can be simplified and used in quasi-steady runs.

\section{RESULTS}

Since experimental data for solid propellant rocket motors is seldom available, we show two representative grain configurations that can verify the merits of the method by allowing for comparisons with results obtained from theoretical calculations using the nozzle theory. All necessary data and equations are provided to assist an interested reader in using the presented problems as benchmarks for independent comparisons. The running time of the computations is of the order of hours on the personal computer.

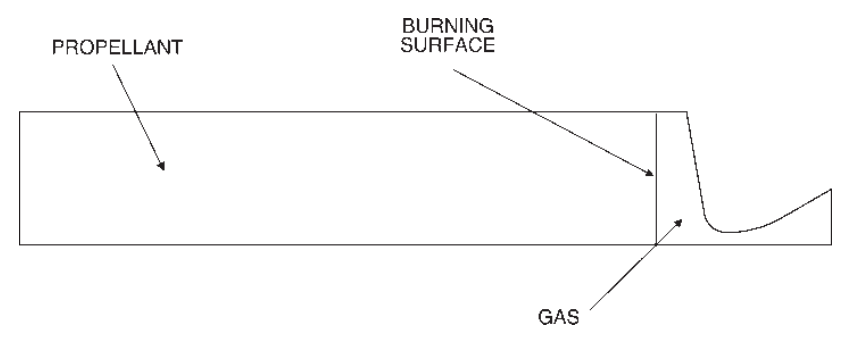

Fig. 6 Cigarette burner configuration. Half of the axisymmetric configuration is shown

First, a cigarette burner rocket motor configuration is considered. The typical geometry is shown in Fig. 6. The cylindrical $0.36 \mathrm{~m}$ diameter motor is $0.9 \mathrm{~m}$ long. The case thickness is neglected. The solid propellant surface is placed at a distance of $0.364 \mathrm{~m}$ from the exit plane of the nozzle. The nozzle profile was constructed as a blend of two circles using inlet diameter of $0.36 \mathrm{~m}$, inlet cone angle of $80^{\circ}$, inlet radius of curvature $0.03 \mathrm{~m}$, exit diameter $0.11 \mathrm{~m}$, exit cone angle $30^{\circ}$, exit radius curvature is $0.15 \mathrm{~m}$ and throat diameter of $0.03 \mathrm{~m}$, giving the length of the nozzle of $0.1637 \mathrm{~m}$. The physical burning surface area and the throat area are therefore $A_{\mathrm{s}}=0.1017876 \mathrm{~m}^{2}$ and $A_{\mathrm{t}}=7.068 \times 10^{-4} \mathrm{~m}^{2}$, respectively.

The propellant and gas data is as follows:

(a) propellant density: $1650 \mathrm{~kg} / \mathrm{m}^{3}$;

(b) reference burning rate: $0.0216 \mathrm{~m} / \mathrm{s}$ at $7 \mathrm{MPa}$;

(c) burning rate exponent: 0.3;

(d) characteristic velocity $c^{*}: 1600 \mathrm{~m} / \mathrm{s}$;

$$
c^{*}=\sqrt{\frac{R T}{\gamma}\left(\frac{\gamma+1}{2}\right)^{(\gamma+1) /(\gamma-1)}}
$$

(e) specific heat ratio $\gamma: 1.2$; 
(f) combustion gases gas constant $R: 370 \mathrm{~J} /(\mathrm{kg} \mathrm{K})$;

(g) static temperature: $2912.77 \mathrm{~K}$;

(h) atmospheric pressure: $0.10 \mathrm{MPa}$;

(i) nozzle exit pressure: $0.078 \mathrm{MPa}$;

(j) nozzle exit temperature $1325.70 \mathrm{~K}$.

In the considered cigarette burner configuration the burning surface area $A_{\mathrm{s}}$ remains constant until the burn-out. Therefore, it is possible to estimate pressure history using a theoretical prediction. The following mass balance equation can be derived form the conservation laws (1) to (3)for isentropic flow.

$$
\frac{\mathrm{d} p_{\mathrm{c}}}{\mathrm{d} t}=\frac{r_{\mathrm{b}} A_{\mathrm{s}}}{V_{\mathrm{g}}}\left(\rho_{\mathrm{s}} R T-p_{\mathrm{c}}\right)-\frac{p_{\mathrm{c}} A_{\mathrm{t}}}{c^{*}} \frac{R T}{V_{\mathrm{g}}}
$$

where $V_{\mathrm{g}}$ is the volume of gas contained within the rocket motor. Equation (12) is an ordinary differential equation that can be readily integrated numerically.

The derivation assumes that the flow is isentropic, static pressure and static temperature in the combustion chamber are homogenous and that the flow in the nozzle is quasi-steady. In general, the above assumptions are not satisfied. Furthermore, the volume of gas $V_{\mathrm{g}}$ increases during the burning process. Nevertheless, equation (12) can be used as an approximation, since during a short time before the equilibrium is reached the changes are small. Similarly, the approximation that $V_{\mathrm{g}}=$ const. is made. This is adequate as for the purpose of comparison we present the initial pressure history until the equilibrium is reached.

A comparison between numerical and theoretical (from (12)) results is shown in Fig. 7. The pressure history was monitored for $0.25 \mathrm{~s}$, at the sensor

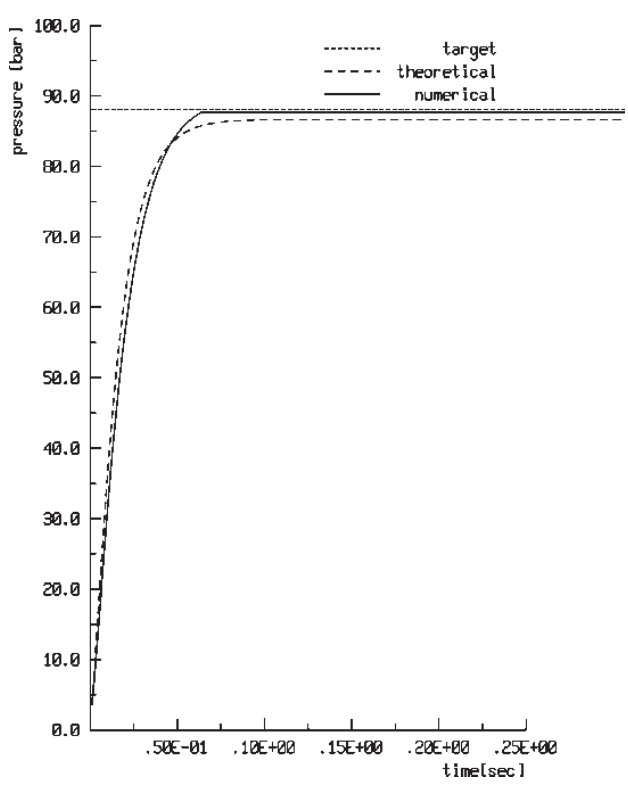

Fig. 7 Pressure history: a comparison between computed and theoretical results positioned at the symmetry axis in the chamber at $0.3 \mathrm{~m}$ from the nozzle exit. Both the slope and the values of the pressure curve are in good agreement, with numerical prediction performing slightly better as it incorporates two-dimensional effects. In particular it computes more realistic (less smoothed) transition to the equilibrium flow. Also the result for the equilibrium pressure of $8.785 \mathrm{MPa}$ obtained by the numerical computation is very close to the target equilibrium pressure obtained from (13) below of $8.792 \mathrm{MPa}$ for this configuration. The equilibrium pressure calculated from (12) is slightly lower and equals $8.731 \mathrm{MPa}$. Pressure histories for several, different points (transducers), placed in the combustion chamber away from the nozzle were also monitored. The results differed at the third decimal point when compared in $\mathrm{MPa}$, with higher values recorded in positions closer to the burning surface. This is consistent with the physics of the problem.

Mesh sensitivity studies for several configurations indicated that in the presented method the accuracy is sensitive to the quality of the mesh at the throat. For inviscid calculations a minimum of eight to ten computational points across the throat is recommended. The details of the computational mesh in the throat area used for the cigarette burner configuration, with ten points across the throat are shown in Fig. 8 . The overall number of nodes in the mesh used for calculation is 25116 .

The second configuration is the axisymmetric geometry shown in Fig. 1. The motor is $0.526 \mathrm{~m}$ long. The outer and inner diameters of the charge are $0.142 \mathrm{~m}$ and $0.060 \mathrm{~m}$, respectively. The charge is bonded to the casing over the length of $0.300 \mathrm{~m}$ starting at the distance of $0.176 \mathrm{~m}$ form the exit plane of the nozzle. The length of the charge decreases symmetrically towards the axis of symmetry at the angle of $55^{\circ}$. The nozzle of the motor is $0.126 \mathrm{~m}$ long and it is constructed as a blend of two circles of the same radii $0.025 \mathrm{~m}$ with inlet slope of $60^{\circ}$ and the outlet slope of $30^{\circ}$. Inlet and exit diameters of the nozzle are $0.142 \mathrm{~m}$ and $0.140 \mathrm{~m}$, respectively and the throat diameter is $0.05 \mathrm{~m}$.

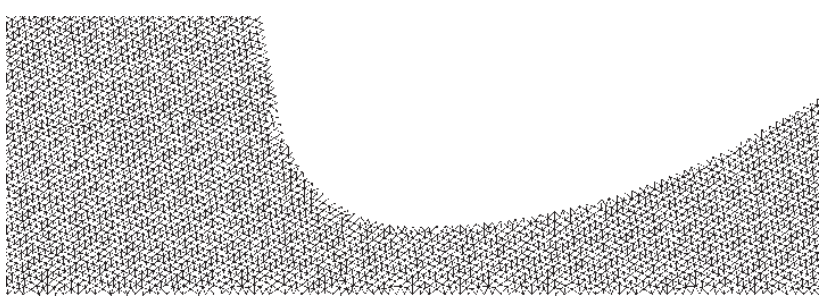

Fig. 8 Mesh quality at the throat. Cigarette burner configuration 
The propellant and gas data is as follows:

(a) propellant density: $1650 \mathrm{~kg} / \mathrm{m}^{3}$;

(b) reference burning rate: $0.020 \mathrm{~m} / \mathrm{s}$ at $7 \mathrm{MPa}$;

(c) burning rate exponent: 0.3;

(d) characteristic velocity $c^{*}: 1425 \mathrm{~m} / \mathrm{s}$;

(e) specific heat ratio $\gamma: 1.2$;

(f) combustion gases gas constant $R: 294.51 \mathrm{~J} /(\mathrm{kg} \mathrm{K})$;

(g) static temperature: $2900.00 \mathrm{~K}$;

(h) atmospheric pressure: $0.1000 \mathrm{MPa}$;

(i) nozzle exit pressure: $0.2160 \mathrm{MPa}$;

(j) nozzle exit temperature $1595 \mathrm{~K}$.

In this case the charge is designed to maintain constant thrust during the operation of the motor and changes in the area of the burning surface are small. Equation (12) is no longer easy to use without information about the changing of volume $V_{\mathrm{g}}$ in time. However, the value of equilibrium pressure at the plateau can be estimated from the equilibrium of mass rates for gases generated in the combustion chamber and gases leaving the nozzle

$$
p_{\mathrm{c}}=\frac{r_{\mathrm{b}} A_{\mathrm{s}} \rho_{\mathrm{s}} c^{*}}{A_{\mathrm{t}}}
$$

By using in equation (13) the value of initial burning surface area $A_{\mathrm{s}}=0.079167 \mathrm{~m}^{2}$ for the given data, the equilibrium pressure is $7.849 \mathrm{MPa}$. Using the maximum burning area recorded during the numerical calculation $A_{\mathrm{s}} \max =0.082842 \mathrm{~m}^{2}$ results

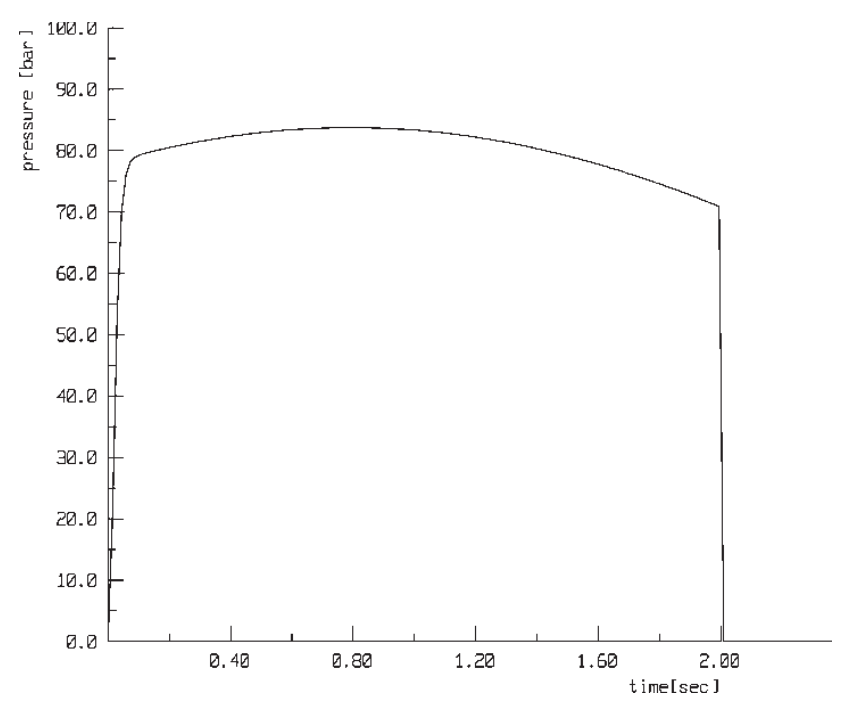

Fig. 9 Pressure history

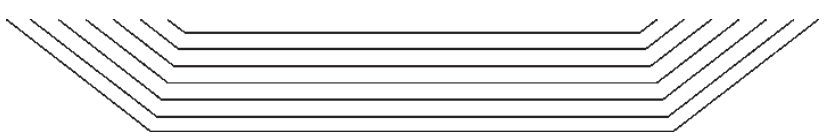

Fig. 10 Burning surface history - half of a cut through the shapes is shown

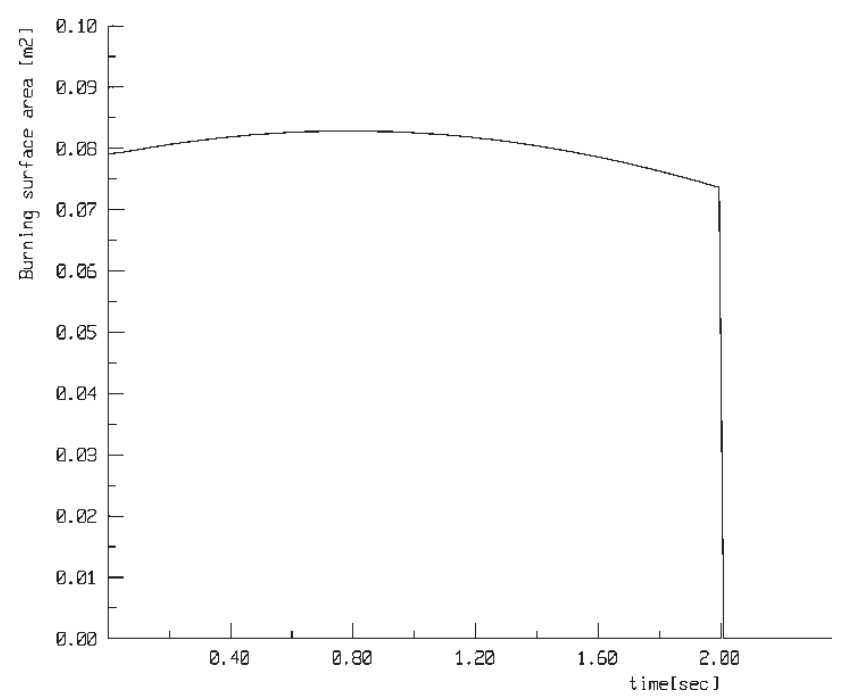

Fig. 11 Burning surface history

in the equilibrium pressure equal to $8.3748 \mathrm{MPa}$. This compares very well with the maximum pressure of 8.3738 MPa recorded during numerical calculation. Figure 9 shows a full pressure history monitored at the centre of combustion chamber. As in the previous examples there was a very minor difference between pressures monitored in several points positioned in the combustion chamber.

Figure 10 presents the axisymmetric cut through the shapes of the burning surface recorded at seven time intervals and Fig. 11 shows the history of the changes in the burning surface area. The computational mesh consisted of 17941 nodes.

\section{CONCLUSIONS}

In this paper a methodology for a unified solution of the solid and gas phases in the modelling of combustion of solids has been proposed. The methodology includes a novel combination of locally moving and updating meshes that allows for tracing changes of burning surface. This technique is general and is particularly suitable for applications with moving and deforming boundaries, which separate computational domains of different materials. The initial study was focused on the gas phase. Further capabilities, such as implementation of the full dynamic solution of both phases and generalization of the method to three-dimensional problems form the natural next step of this development. The method appears to be particularly suitable for problems with moving and deforming boundaries separating several computational domains, and can be employed for a variety of applications for which traditionally modelling is conducted independently for 
each computational domain and then coupled on the boundaries.

\section{ACKNOWLEDGEMENTS}

This work was partially supported by the Royal Society of London travel grant No. 15048. The authors are grateful to Professor Anna B. Crowley for her comments and helpful discussions.

\section{REFERENCES}

1 Gallier, S. and Guery, J. F. State of the art of CFD for solid rocket motors. QNET-CFD Netw. Newsl., 2001, 1(2), 21-24.

2 Le Breton, P., Ribereau, D., Godfroy, F., Abgrall, R., and Augoula, S. SMR performance analysis by coupling burnback and pressure field computations. AIAA paper 98-3968, 1998.

3 Le Breton, P., Guert, J. F., Vuillot, F., and Prevost, M. Recent advances in the prediction of SMR thrust oscillations. Premier Colloque Europeen sur la Technologie des Lanceurs "Vibration des Lanceurs", Toulouse, France, 14 December 1999.

4 Fiedler, R., Breitenfeld, M., Jiao, X., Haselbacher, A., Gebelle, P., Guoy, D., and Brandyberry, M. Simulations of slumping propellant and flexing inhibitors in solid rocket motors. 38th AIAA/ASME/SAE/ASEE JPC, Indianapolis, IN, July 2002, 2002-4341.

5 Fiedler, R., Jiao, X., Namazifrad, A., Haselbacher, A., Najjar, F., and Parsons, I. Coupled fluid-structure 3D solid rocket motor simulations. 37th AIAA/ASME/SAE/ASEE JPC Conf. and Exhib., Salt Lake City, UT, July 2001, 2001-3954.

6 Parsons, I., Alavilli, P., Namazifard, A., Acharyja, A., Jiao, X., and Fiedler, R. Coupled simulations of solid rocket motors. AIAA paper 2000-3456, 2000.

7 Barth, T. Aspects of unstructured grids and finite volume solvers for the Euler and Navier-Stokes equations. In Special Course on Unstructured Grid Methods for Advection Dominated Flows, AGARD Report 787, 1992:6.1-6.61 1992.

8 Kuo, K. Principles of combustion, 1986 (John Wiley \& Sons, Chichester, UK).

9 Smolarkiewicz, P. K. and Szmelter, J. MPDATA: An edge based unstructured-grid formulation. J. Comput. Phys., 2005, 206, 624-649.

10 Luo, H., Baum, J., and Lohner, R. High-Reynolds number viscous flow computations using an unstructuredgrid method. AIAA paper 2004-1103, 2004.

11 Jameson, A., Schmidt, W., and Turkel, E. Numerical solution of the Euler equations by finite volume methods using Runge-Kutta time-stepping schemes. AIAA paper 81-1259, 1981.

12 Zang, H., Reggio, M., Trepanier, J., and Camarero, R. Discrete form of the GCL for moving meshes and its implementation in CFD schemes. Comput. Fluids, 1993, 22(1), 9-23.

13 Mombelli, C., Guichard, A., Godfroy, F., and Guery, J. F. Parallel computation of vortex-shedding in solid rocket motors. AIAA paper 99-2510, 1999.
14 Anthoine, J., Buchlin, J. M., and Guery, J. F. Experimental and numerical investigations of nozzle geometry effect on the instabilities in solid propellanr boosters. AIAA paper 2000-3560, 2000.

15 Sutton, G. and Ross, M. Rocket propulsion elements. An introduction to the engineering of rockets, 1976 (John Wiley \& Sons, Chichester, UK).

16 Lohner, R., Morgan, K., and Zienkiewicz, O. C. An adaptive finite element procedure for high speed flows. Comput. Methods. Appl. Mech. Eng., 1985, 51, 441-465.

17 Lohner, R. Applied CFD techniques. An introduction based on finite element methods, 2001 (John Wiley \& Sons, Chichester, UK).

18 Batina, J. Unsteady Euler airfoil solutions using unstructured dynamic meshes. AIAA J., 1990, 28(8), 1381-1388.

19 Blom, F. J. Considerations on the spring analogy. Int. J. Numer. Methods Fluids, 2000, 32, 647-668.

20 Yang, Z. and Mavriplis, D. Unstructured dynamic meshes with higher-order time integration schemes for the unsteady Navier-Stokes equations, AIAA paper 2005-1222, 2005.

\section{APPENDIX}

\begin{tabular}{ll} 
Notation & \\
$A_{\mathrm{s}}$ & total burning propellant area \\
$A_{\mathrm{t}}$ & nozzle throat area \\
$C_{\mathrm{p}}$ & specific heat at constant pressure \\
$c^{*}$ & characteristic velocity \\
$\mathrm{d} S$ & element of boundary $S$ \\
$\mathrm{~d} V$ & element of volume $V$ \\
$\boldsymbol{D}$ & artificial dissipation terms vector \\
$E$ & total specific energy \\
$\boldsymbol{F}$ & fluxes vector \\
$i, j$ & mesh vertexes \\
$\boldsymbol{I}$ & unit tensor \\
$k$ & thermal conductivity \\
$n$ & burning rate pressure exponent \\
$\boldsymbol{n}$ & unit normal vector \\
$p$ & pressure \\
$p_{\mathrm{c}}$ & combustion pressure \\
$p_{\mathrm{ref}}$ & reference pressure \\
$\boldsymbol{Q}$ & source vector \\
$r$ & radial coordinate \\
$r_{\mathrm{b}}$ & propellant burning rate \\
$r_{\text {ref }}$ & reference burning rate \\
$R$ & gas constant \\
$S$ & boundary or surface area of the \\
$t$ & computational domain/volume \\
$T$ & time \\
$T_{\mathrm{f}}$ & static temperature \\
$\boldsymbol{u}$ & isochoric flame temperature \\
$V$ & velocity vector \\
$\boldsymbol{w}$ & volume \\
$x$ & interface/mesh velocity vector \\
& axial coordinate \\
& \\
\hline &
\end{tabular}


$\alpha$

method

$\gamma \quad$ specific heat ratio

$\delta t \quad$ time increment

$\rho \quad$ density of the material

$\sigma \quad$ stress tensor

$\boldsymbol{\Psi}_{i} \quad$ discretized variables vector
Subscripts

b burning

g gas

$r$ radial

$\mathrm{s} \quad$ solid

$x \quad$ axial 
